Pacific Journal of Mathematics

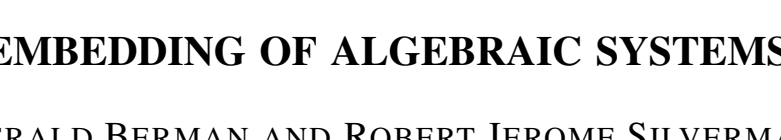




\section{EMBEDDING OF ALGEBRAIC SYSTEMS}

\section{Gerald Berman and Robert J. Silverman}

1. Introduction. The representation of semi-groups and algebras by systems of transformations has proved useful in algebra. This paper develops a representation theory for systems with an arbitrary number of algebraic operations by systems of transformations. Among the systems considered will be systems having $n$ binary compositions $t_{1},+_{2}, \cdots, t_{n}$, such that $(A),{ }_{{ }_{i}}$ is associative, $i=1,2, \cdots, n,(B),{ }_{+}{ }_{j}$ is left distributive with respect to $+_{i}$ for $0<i<j, j=2, \cdots, n$. Many of the well known algebraic systems are of this type, e.g., semi-groups, groups, rings, near-rings, and semi-rings.

It is shown that any system satisfying conditions $(A)$ and $(B)$ can be embedded isomorphically into a system whose elements are transformations on a set, and whose binary compositions are either operator multiplication or derived from operator multiplication in a simple way. It is noted that this transformation system is completely characterized by a set, and the theory is a natural generalization of the corresponding theory for groups. Further, these transformation systems may have the additional property that the elements of the isomorphic image are right multiplication operators relative to operator multiplication on other systems.

The systems considered are generalized to include systems containing operations which need not satisfy $(A)$ or $(B)$ or be defined everywhere. An example of such a system is a neo-ring (defined in $\S 5$ ). Embedding theorems into transformations (in particular into right multiplications) are proved for such systems, and, as in the previous case, the binary compositions having properties $(A)$ and $(B)$ are identified with operator multiplication. Generalizations of modules, operator groups, and algebras are also considered and the corresponding embedding theorems indicated.

The theory developed subsumes many of the well known embedding theorems. For example, a ring is embedded isomorphically into a ring of transformations (endomorphisms). This is done without first embedding into a ring with identity.

In $\S 2$ algebraic systems are defined, notation is introduced, and some examples are presented. The transformation systems are considered in $\S 3$. They are used to show the existence of the systems defined in $\S 2$, and some of their properties are developed. Section 4 contains the main embedding theorem which asserts that any one of the systems defined in $\S 2$ is isomorphic to a subsystem of a transformation system as defined in $\S 3$. Applications of this embedding theorem are made in

Received October 28, 1958. 
$\S 5$ to algebraic systems with two binary compositions. In $\S 6$ the systems which generalize operator systems are discussed.

2. Definitions and examples. A semi-composition on a non-empty set $S$ is a mapping from a non-empty subset of $S \times S$ to $S$. A composition (binary composition) on a set $S$ is a semi-composition whose domain is $S \times S$. A $t$-polyoid is an algebraic system $S=\left(S,+_{1},+_{2}, \cdots,+_{t}\right)$, where $S$ is a non-empty set and $+_{i}$ is a semi-composition on $S, i=1,2, \cdots, t$. Denote the domain of $+_{i}$ by $S_{i} \subset S \times S$, and denote the image of $(a, b) \in S_{i}$ by $a+{ }_{i} b$. A 0 -polyoid is a non-empty set $S$.

A semi-composition $t_{2}$ is left distributive with respect to a semicomposition $+_{1}$ if $a+{ }_{2}\left(b+{ }_{1} c\right)$ is defined if and only if $\left(a+{ }_{2} b\right)+{ }_{1}\left(a+{ }_{2} c\right)$ is defined, and the two expressions are equal. A semi-composition + is associative if, whenever $a+b$ and $b+c$ are defined, then $(a+b)+c$ is defined if and only if $a+(b+c)$ is defined and $(a+b)+c=a+(b+c)$. A semi-composition + is commutative if $a+b$ is defined if and only if $b+a$ is defined and $a+b=b+a$.

A $(t, n)$-polyring is a $(t+n)$-polyoid $\subseteq=\left(S,+_{1},+_{2}, \cdots,+_{t+n}\right)$ such that

(i ) $\left(S,+_{t+j}\right)$ is a semi-group, $j=1,2, \cdots, n$, and

(ii) $+{ }_{j}$ is left distributive with respect to ${ }_{+}$for $1 \leqq i<j, t+n \geqq j>t$. An $n$-polyring is a $(0, n)$-polyring.

A semi-group is a 1-polyring. Rings, near-rings and semi-rings are 2-polyrings. An associative left neo-ring $(\S 5)$ is a $(1,1)$-polyring, but not a 2-polyring. Further realizations of $(t, n)$-polyrings will be indicated. In fact, it will be shown that any $t$-polyoid can be embedded nontrivially into a $(t, n)$-polyring for any positive integer $n$.

A loop is an example of a polyoid which is not a polyring. Operator groups can also be considered as 2-polyoids if $S$ is taken as $M \cup G$, where $M$ is the set of operators, and $G$ is the set of elements of the group. (If the group operation is denoted by $t_{1}$, and if $t_{2}$ is the operation which applies an operator to a group element, then the domain of $+_{1}$ is $S_{1}=G \times G$, and the domain of $+_{2}$ is $\left.M \times G\right)$. Lie, Jordan, alternative, and other non-associative rings are also examples of 2-polyoids which are not polyrings. A well known example of a 3-polyring (with right distributive law instead of left) is the set of real-valued functions over the real numbers, the operations being point-wise addition, pointwise multiplication, and functional substitution. Vector spaces, and in general, modules, can be considered as 4-polyoids.

A mapping $\phi$ from $S$ to $S^{\prime}$ is a homomorphism from a 1-polyoid $\mathfrak{S}=(S,+)$ to a 1 -polyoid $\mathfrak{S}^{\prime}=\left(S^{\prime},+^{\prime}\right)$, if, whenever $a+b$ is defined then $(a \phi)+^{\prime}(b \phi)$ is defined and $(a+b) \phi=(a \phi)+{ }^{\prime}(b \phi)$. The mapping $\phi$ is an isomorphism if in addition

(i ) $\phi$ is a $1-1 \mathrm{map}$, and 
(ii) if $(a \phi)+^{\prime}(b \phi)$ is defined, then $a+b$ is defined. If, further, $\phi$ is onto, then $\subseteq$ is isomorphic to $\mathfrak{S}^{\prime}$. A mapping $\phi$ from a $t$-polyoid $\mathfrak{S}=\left(S ;+_{1},+_{2}, \cdots,+{ }_{\imath}\right)$ to a $t$-polyoid $\mathfrak{S}^{\prime}=\left(S^{\prime},+_{1}^{\prime},+_{2}^{\prime}, \cdots,+_{t}^{\prime}\right)$ is an (ordered) homomorphism if $\phi$ is a homomorphism from $\left(S,+_{i}\right)$ to $\left(S^{\prime},+_{i}^{\prime}\right), i=1,2, \cdots$, t. Analogously $\phi$ is an isomorphism if $\phi$ is an isomorphism from $\left(S,+_{i}\right)$ to $\left(S^{\prime},+_{1}^{\prime}\right), i=1,2, \cdots, t$. A 1-polyoid $\left(R,+^{\prime}\right)$ is a subsystem of a 1-polyoid $(S,+)$ if $R \subset S$ and the identity map on $R$ is an isomorphism from $\left(R,+^{\prime}\right)$ into $(S,+)$. An $r$-polyoid $\left(S,+_{1},+_{2}, \cdots,+_{r}\right)$ is a subsystem of a $t$-polyoid $\left(S^{\prime},+{ }_{1}^{\prime},+_{2}^{\prime}, \cdots,+{ }_{t}^{\prime}\right)$ if $S \subset S^{\prime}$ and if there exists a $1-1$ correspondence between $+_{i}$ and $+_{j_{i}}^{\prime}, i=1,2, \cdots, r$, such that $\left(S,+_{i}\right)$ is a subsystem of $\left(S^{\prime},+^{\prime}{ }_{j}\right)$.

3. Transformation polyrings. The theorem of this section asserts that $(t, n)$-polyrings exist, and in fact can be realized in terms of systems of transformations, where a composition which is associative and left distributive is either operator multiplication or derived from operator multiplication by pointwise addition corresponding to operator multiplication defined on underlying sets of transformations. These particular systems are important in that any $(t, n)$-polyring is contained isomorphicly as a subsystem. This will be shown in $\S 4$. In the process of constructing the transformation systems it is shown that any $(t, n)$-polyring can be embedded properly into a larger system. However, relative to the corresponding isomorphism the compositions cannot be identified with operator multiplication.

Let $\subseteq=\left(S,+{ }_{1},+_{2}, \cdots,+_{t}\right)$ be a $t$-polyoid such that $S$ contains more than one element. Define $\sum(0, \mathfrak{S})=\mathfrak{S}$, and let $S^{0}=S,+{ }_{i}^{0}=+_{i}$, $i=1,2, \cdots, t$. Further define $\sum(j, \varrho)=\left(S^{j},{ }_{1}^{j},+{ }_{2}^{j}, \cdots,+{ }_{t}^{j}, \circ_{1}^{j}, \circ_{2}^{j}, \cdots, \circ_{j}^{j}\right)$, $j=1,2, \cdots$, where, inductively $S^{j}$ is the set of transformations on $S^{j-1}$, $j=1,2, \cdots$, and $+_{i}^{1}$ is point-wise addition in $S^{1}$ corresponding to $+_{i}$, $i=1,2, \cdots, t$, i.e. $s\left(a+{ }_{i}^{1} b\right)=(s a)+{ }_{i}(s b), s \in S, a, b \in S^{1}$, wherever defined, and inductively $+_{i}^{j}$ is pointwise addition in $S^{j}$ corresponding to $+_{i}^{j-1}$ in $S^{j-1}, i=1,2, \cdots, t ; j=1,2, \cdots$ The composition $\circ_{k}^{k}$ is operator multiplication on $S^{k}$, and $\circ_{k}^{k+1}$ is point-wise addition in $S^{k+1}$ corresponding to $\circ_{k}^{k}$. Inductively $\circ_{k}^{j}$ is point-wise addition in $S^{j}$ corresponding to $o_{k}^{j-1}$ in $S^{j-1}$, for $j>k$. The system $\sum(j, \subseteq)$ will be called a $(t, j)$-transformation polyring, $j=1,2, \cdots$

THEOREM 1. A $(t, j)$-transformation polyring is a $(t, j)$-polyring. Further, $\Sigma(j, \mathfrak{S})$ contains a subsystem isomorphic to $\Sigma(k, \mathfrak{S})$ for $k=1,2, \cdots, j$.

The following lemmas from which the theorem follows are easily verified.

Lemma 1. Point-wise addition is a semi-composition and is a (associative, commutative) composition if and only if it is derived from 
$a$ (associative, commutative) composition.

Lemma 2. The semi-composition $+_{i}$ is (right) left distributive with respect to ${ }_{+}{ }_{j}$ if and only if the semi-composition $+_{i}^{k}$ is (right) left distributive with respect to $+_{j}^{k}$.

Lemma 3. The composition operator multiplication in $S^{j}$ is left distributive with respect to any point-wise addition defined in $S^{j}$.

Let $\mu^{j}$ be the mapping from $S^{j}$ to $S^{j+1}, j=0,1, \ldots$ defined by $a \mu^{\jmath}=x_{a}$, where $b x_{a}=a, a, b \in S^{j}$.

Lemma 4. The system $\left(S^{j} \mu^{j},{ }_{+}^{j+1}\right)$ is isomorphic to $\left(S^{j},{ }_{+}^{j}\right)$ relative to $\mu^{3}$.

The system $\sum(1, \mathfrak{S})$ is a $(t+1)$-polyoid by Lemma 1 , a $(t, 1)$-polyring by Lemma 3 , and $\subseteq$ is isomorphic to a subsystem of $\sum(1, \mathfrak{S})$ by Lemma 4. The theorem follows by induction making use of Lemma 3 to go from $\sum(j-1, \mathfrak{S})$ to $\Sigma(j, \mathfrak{S})$. The isomorphism from $\sum(k, \mathfrak{D})$ into $\Sigma(j, \mathfrak{S}), j>k$, is $\mu^{k} \mu^{k+1} \cdots \mu^{j-1}$. The following corollary is immediate.

COROLlaRy. (i) For any integer $n$, there exists an n-polyring such that the $n$ compositions are distinct.

(ii) Given any $(t, n)$-polyring $\subseteq$ and any positive integer $m$, there exists $a(t, n+m)$-polyring $\mathfrak{I}$ containing a subsystem $\mathfrak{S}^{\prime}$ which is a $(t, n)$-polyring isomorphic to $\mathfrak{S}$.

(iii) Corresponding to any $(t, n)$-polyring $\subseteq$ with underlying set $S$, there exists $a(t, n)$-polyring $\mathfrak{I}$ with underlying set $T$ having cardinality greater than the cardinality of $S$ such that $\mathfrak{I}$ contains an isomorphic image of $\mathfrak{S}$.

The isomorphism $\mu^{j}$ is not the main embedding discussed in this paper. The primary use made of this mapping is to show that the systems considered can be embedded into larger systems. Not only is the cardinality of the underlying set larger, but additional compositions are introduced, and the semi-compositions corresponding to those in $S$ are defined over a larger domain than the isomorphic image.

The domain of $+_{i}^{j+1}$ (the point-wise addition in $S^{j+1}$ corresponding to ${ }_{t_{i}}$ in $S$ ) contains pairs whose components are not in $S^{j} \mu^{j}$, the image of $S$, provided only that there exist two pairs of elements in $S,(a, b) \neq(c, d)$, such that $a+{ }_{i} b, c+{ }_{i} d$ are defined. This implies that $\subseteq$ is embeddable non-trivially into a larger system. To see this, partition $S$ into two non-empty subsets $A$ and $B$. Define $a^{\prime}, b^{\prime} \in S^{1}: f a^{\prime}=a, f \in A, f a^{\prime}=c$, $f \in B ; f b^{\prime}=b, f \in A, f b^{\prime}=d, f \in B$. Then $a^{\prime}+b^{\prime}$ is defined and is not in the isomorphic image of $S$. The general result is immediate by induction.

A few of the properties of point-wise addition, some of them well known, are now presented. 
The following properties of $\subseteq$, which are carried over to the isomorphic image, are also properties of the larger system $\Sigma(j, \mathfrak{S})$.

(a) The semi-composition $+_{i}$ is

(1) a composition,

(2) associative,

(3) commutative.

(b) (1) There exists an (unique) identity $e_{i}$ (right, left, two-sided) relative to $+_{i}$ in $\left(S,+_{i}\right)$.

(2) Every element of $S$ has an inverse (right, left, two-sided) relative to $+_{i}$ and $e_{i}$.

(c) The right equation $a+{ }_{i} x=b$ has a (unique) solution $x \in S$ for every $a, b \in S$ (A similar statement holds for left equations).

(d) The system $\left(S,+_{i}\right)$ is a group (loop, semi-group, semi-loop, i.e. $+_{i}$ is a composition). to $+_{j}$.

(e ) The semi-composition $+_{i}$ is left (right) distributive with respect

(f) The system $\left(S,+_{i},+_{j}\right)$ is a ring (near-ring, semi-ring, neo-ring).

$(\mathrm{g})$ The system $\subseteq$ is an $(r, m)$-polyring.

(h) If there exists an $a_{i}$ in $S$ such that $a_{i}+{ }_{i} b=c+a_{i}=a_{i}$ whenever the sums are defined and there exist $b, c \in S$ such that the sums are defined, and if the image of $a_{i}$ under the embedding is $a_{i}^{j} \in S^{j}$, then $a+{ }_{i}^{j} a_{i}^{j}=a_{i}^{j}+{ }_{i}^{j} a=a_{i}^{j}$, whenever the sums are defined.

However, some of the properties of $\subseteq$ are not carried over to the whole of $\sum(j, \mathfrak{S})$. For example, referring to (h): There exist elements $b, c \in S^{j}, b, c \neq a_{i}^{j}$ such that $b+{ }_{i}^{j} c=a_{i}^{j}$. This property is a generalization of divisors of zero. The elements $a_{i}$, $a_{i}^{j}$ behave like an additive zero relative to the multiplication in a ring. Thus, the system $\left(S_{i}^{j},+_{i}^{j},{ }^{j}{ }_{k}^{j}\right)$, $j=1,2, \cdots$, is not a division ring (integral domain, near-field, left or right neo-field) even though $\left(S,+_{i},+_{k}\right)$ is such a system.

4. The embedding theorem. In this section it is shown that any $(t, n)$-polyring can be embedded into a transformation polyring $\Sigma(j, \mathfrak{S})$, where the associative and distributive compositions are associated with operator multiplication on sets of transformations. Further the elements of the isomorphic image can be identified with right multiplications relative to operator multiplication on an underlying set of transformations. The method of proof is different from the usual one for rings in that the embedding is accomplished without first adjoining an identiy to the system.

Let $\mathfrak{S}=\left(S,+_{1},+_{2}, \cdots,+_{t+n}\right)$ be a $(t, n)$-polyring. An isomorphism from $\mathfrak{S}$ into the transformation polyring $\sum(m, \mathfrak{I})=\left(T^{m},+_{1}^{m},+_{2}^{m}, \cdots\right.$, $\left.+_{t}^{m}, \circ_{1}^{m}, \circ_{2}^{m}, \cdots, \circ_{m}^{m}\right)$ is a natural isomorphism if $+_{t+i}$ corresponds to $\circ_{j_{i}}^{m}, i=1,2, \cdots, n$, where $j_{1}<j_{2}<\cdots<j_{n} \leq m$. 
THEOREM 2. There exists a natural isomorphism from a $(t, n)$ polyring to a transformation polyring $\Sigma(m, \mathfrak{I})$ for some $t$-polyoid $\mathfrak{I}$, $m \geq n$. Further if $m>n$, the elements of the isomorphic image can be taken as right translation operators relative to operator multiplication in $T^{m-1}$.

By virtue of the corollary of Theorem $1(\S 3)$ there exists a $(t, n)$ polyring $\mathfrak{T}=\left(T,+{ }_{1}^{\prime},{ }_{2}^{\prime}, \cdots,+_{t+n}^{\prime}\right)$ containing an isomorphic image of $\mathfrak{S}$ and such that the cardinality of $T$ is greater than the cardinality of S. Let $\mathfrak{S}_{\jmath}=\left(S,+_{1},+_{2}, \cdots,+_{t+j}\right)$.

Lemma 1. Given a 1-polyoid $(A,+)$, a set $B$ and $a 1-1$ map $\phi$ of $A$ into $B$, then there exists a semi-composition ${ }^{+}$on $A \phi$ such that $\left(A \phi,+^{\prime}\right)$ is isomorphic to $(A,+)$ under $\phi$.

It can easily be checked that the semi-composition ${ }^{+}{ }^{\prime}$ defined by $b_{1}+{ }^{\prime} b_{2}=\left(a_{1}+a_{2}\right) \phi$, where $a_{1} \phi=b_{1}, a_{2} \phi=b_{2}$, whenever $a_{1}+a_{2}$ is defined, has the required property.

LEMMA 2. There exists a natural isomorphism from $\mathfrak{S}_{j}$ into $\Sigma(j, \mathfrak{T})$.

Identify $\mathfrak{S}$ with its image in $\mathfrak{T}$, and for convenience set $+_{i}^{\prime}=+_{\imath}$, $i=1,2, \cdots, t+n$. Let $\phi_{1}$ be the map from $S$ to $T^{1}$ defined by $a\left(s \phi_{1}\right)=a+_{t+1} s, a \in S, a\left(s \phi_{1}\right)=s, a \in T, a \notin S$, for $s \in S$. Let $+_{i}^{1}$ be defined as point-wise addition in $T^{1}$ corresponding to $+_{i}$ in $T, i=$ $1,2, \cdots, t$. The system $\left(S \phi_{1},+{ }_{1}^{1},+{ }_{2}^{1}, \cdots,+{ }_{t}^{1}, \circ_{1}^{1}\right) \subset \sum(1, \mathfrak{T})$ (where $\circ_{1}^{1}$ is operator multiplication in $T^{1}$ ) is isomorphic to $\mathfrak{S}_{1}$. The map $\phi_{1}$ is $1-1$, for if $a \phi_{1}=b \phi_{1}$, then, for $c \in T, c \notin S, a=c\left(a \phi_{1}\right)=c\left(b \phi_{1}\right)=b$. The map $\phi_{1}$ is a homomorphism from $\left(S,+_{\imath}\right)$ to $\left(S \phi_{1},+_{i}^{1}\right), i=1,2, \cdots, t$, since if $a+{ }_{i} b$ is defined, then, for $c \in S, a\left(\left(a+{ }_{i} b\right) \phi_{1}\right)=c+{ }_{t+1}\left(a+{ }_{i} b\right)=$ $\left(c+_{t+1} a\right)+{ }_{i}\left(c+_{t+1} b\right)=c\left(a \phi_{1}\right)+{ }_{i} c\left(b \phi_{1}\right)=c\left(\left(a_{1} \phi_{1}\right)+{ }_{i}^{1}\left(b \phi_{1}\right)\right)$, and for $c \notin S$, $c \in T, c\left(\left(a+{ }_{i} b\right) \phi_{1}\right)=a+{ }_{i} b=c\left(a \phi_{1}\right)+{ }_{i} c\left(b \phi_{1}\right)=c\left(\left(a \phi_{1}\right)+{ }_{i}^{1}\left(b \phi_{1}\right)\right)$. Further, $a+{ }_{i} b$ is defined if and only if $\left(a \phi_{1}\right)+{ }_{i}^{1}\left(b \phi_{1}\right)$ is defined since for $c \in T$, $c \notin S, c\left(\left(a \phi_{1}\right)+{ }_{i}^{1}\left(b \phi_{1}\right)\right)=a+{ }_{i} b$. The map $\phi_{1}$ is also a homomorphism from $\left(S,+_{t+1}\right)$ to $\left(S \phi, \circ_{1}^{1}\right)$ since for $c \in S, c\left(\left(a+_{t+1} b\right) \phi_{1}\right)=c+_{t+1}\left(a+_{t+1} b\right)=$ $\left(c+_{t+1} a\right)+_{t+1} b=\left(c\left(a \phi_{1}\right)\right)\left(b \phi_{1}\right)=c\left(\left(a \phi_{1}\right) \circ_{1}^{1}\left(b \phi_{1}\right)\right)$, and, for $c \notin S, c \in T$, $c\left(\left(a+_{t+1} b\right) \phi_{1}\right)=a+_{t+1} b=a\left(b \phi_{1}\right)=\left(c\left(a \phi_{1}\right)\right)\left(b \phi_{1}\right)=c\left(\left(a \phi_{1}\right) \circ_{1}^{1}\left(b \phi_{1}\right)\right)$. Thus the lemma holds for $j=1$. The proof follows by induction. Assume the lemma for $j=k$. Let $\phi_{k}$ be the isomorphism from $\mathfrak{S}_{k}$ into $\sum(k, \mathfrak{T})$. Let $\left(S \phi_{k}, \circ_{k+1}^{k}\right)$ be the image of $\left(S,+_{t+k+1}\right)$ as in Lemma 1. Define $\phi_{k+1}$ from $S$ to $T^{k+1}$ by $\phi_{k+1}=\phi_{k} \theta_{k+1}$, where, for $a \in S \phi_{k}, c\left(a \theta_{k+1}\right)=c \circ{ }_{k+1}^{k} a$ for $c \in S \phi_{k}, c\left(a \theta_{k+1}\right)=a$, for $c \in T^{k}, c \notin S \phi_{k}$. As in the case $j=1$, the system $\left(S \phi_{k},+_{1}^{k},+_{2}^{k}, \cdots,+_{t}^{k}, \circ_{1}^{k}, \circ_{2}^{k}, \cdots, \circ_{k+1}^{k}\right)$ is isomorphic to $\left(S \phi_{k+1},+_{1}^{k+1}\right.$, $\left.+{ }_{2}^{k+1}, \cdots,+{ }_{t}^{k+1}, \circ_{1}^{k+1}, \circ_{2}^{k+1}, \cdots, \circ_{k+1}^{k+1}\right) \subset \sum(k+1, \mathfrak{I})$ under $\theta_{k+1}$. Hence $\phi_{k+1}$ is an isomorphism.

LEMma 3. There exists a natural isomorphism from $\mathfrak{S}_{\text {, into }}$ 
$\sum(j+k, \mathfrak{I})$ such that the elements of the isomorphic image are right translations relative to operator multiplication on $T^{j+k-1}, k=1,2, \cdots$

Consider the map $\mu_{j+1}$ from $T^{j}$ to $T^{j+1}$ defined by $b\left(a \mu_{j+1}\right)=b \circ_{j}^{j} a$, $a, b \in T^{j}$, $\left(\circ_{j}^{j}\right.$ is operator multiplication on $\left.T^{j}\right)$. The fact that there is an identity relative to operator multiplication in $T^{j}$ guarantees that $\mu_{j+1}$ is $1-1, j=1,2, \cdots$. Then, as an easy extension of the corresponding theorem in ring theory, $\mu_{j+1}$ is an isomorphism from $\sum(j, \mathfrak{I})$ to $\sum(j+1, \mathfrak{I})$, where the elements of the isomorphic image are right translations relative to ${ }^{j}$. Thus $\phi_{j} \mu_{j+1}$ is an isomorphism satisfying the conditions of Lemma 3 for $k=1$. By using the same construction the Lemma follows by induction.

Theorem 2 can now be proved. By Lemma 2 there exists a natural isomorphism from $\mathfrak{S}$ into $\Sigma(n, \mathfrak{I})$, and Lemma 3 yields a natural isomorphism into $\sum(m, \mathfrak{I}), m>n$. Lemma 3 also insures that for $m>n$ the elements of the isomorphic image are right translations relative to operator multiplication on $T^{m-1}$.

This embedding theorem is an example of a class of embeddings of $(t, n)$-polyrings into transformations. For example, the family of transformation polyrings $\sum(2 i, \mathfrak{I}), i=1,2, \cdots, n$ has the property that, for each $i, i=1,2, \cdots, n$, there exists a natural isomorphism from $\mathfrak{S}_{i}$ into $\sum(2 i, \mathfrak{I})$ such that the elements of the isomorphic image are right translations relative to operator multiplication on $T^{2 i-1}$.

5. Applications. The results of this paper can be applied directly to many of the standard algebraic systems. For example, the well known embedding theorems of groups (semi-groups) and rings into transformation systems follow at once from Theorem 2.

There are other polyrings in the literature to which the embedding theorems apply. For example, near-rings [1], semi-rings [2], and associative neo-rings [3]. An associative left semi-neo-ring $\mathfrak{A}$ is a system $(S,+, \circ)$, where $S$ is a non-empty set, + is a composition, $(S, \circ)$ is a semi-group, and $\circ$ is left distributive with respect to + . An associative left neo-ring $\mathfrak{B}$ is an associative left semi-neo-ring with the property that $(S,+)$ is a loop. An associative neo-ring $\mathbb{C}$ is an associative left neoring in which th operation $\circ$ is right distributive with respect to + . A left semi-ring $\mathfrak{D}$ is an associative left semi-neo-ring with the property that the operation + is associative (i.e. $(S,+)$ is a semi-group). A semiring $\&$ is a left semi-ring with the property that $\circ$ is right distributive with respect to + . A near-ring $\mathfrak{F}$ is a left semi-ring with the property that $(S,+)$ is a group.

Examples of systems $\mathfrak{A}, \mathfrak{B}, \mathfrak{D}$, and $\mathfrak{F}$ are given by the following construction. Consider a system $(R,+)$ which is a semi-loop (i.e., + is a composition), loop, semi-group, or group. Then the system $\left(S,+^{\prime}, \circ\right)$, where $S$ is the set of transformations on $R,+^{\prime}$ is point-wise addition 
on $S$ defined from + in $R$, and $\circ$ is operator multiplication is $S$, is a system of type $\mathfrak{A}, \mathfrak{B}, \mathfrak{D}$, or $\mathfrak{F}$ respectively.

The following corollary to Theorem 2 is immediate.

CoRollary. Any system $\mathfrak{A}, \mathfrak{B}, \mathfrak{C}, \mathfrak{D}, \mathfrak{F}$, or $\mathfrak{F}$ can be embedded isomorphically into the transformations on a semi-loop, loop, loop, semigroup, semi-group, group, respectively, where the second operation (multiplication) is operator multiplication, and the other operation (addition) is point-wise addition. Each of these systems can be embedded isomorphically into a system of the same type with multiplicative identity. Further the isomorphic image can be taken in the right translations relative to operator multiplication defined on an underlying set of transformations. In case the right distributive law holds, the system is embedded into the endomorphisms of the underlying additive system.

Several of the $(1,1)$-polyrings above are 2-polyrings, i.e. rings near-rings, (left-) semi-rings. For such systems stronger statements can be made. For example, there exists a natural isomorphism from any 2-polyring $\mathfrak{S}=(S,+, \cdot)$ to $\Sigma(2, T)$ for some set $T$. (This statement also holds for $n$-polyrings.) It is interesting to note that $\sum(2, T)\left(\sum(n, T)\right)$ is completely determined by the set $T$. In case S contains identities for both compositions (e.g. ring with identity), then $\mathfrak{I}$ can be taken to be $\mathfrak{S}$. The conditions that identities exist can be replaced by the left cancellation law for each composition, or by the condition that for $a \neq b \in S$, there exist elements $c, c^{\prime} \in S$ such that $c+a \neq c+b, c^{\prime} a \neq c^{\prime} b$. In particular fields, division rings, or more generally near-fields, and integral domains can be embedded into $\Sigma(2, S)$, the second transformation polyring on the underlying set $S$.

6. Some generalizations. It is possible to generalize the concept of groups with operators, modules, algebras, and so forth to $n$-polyoids with operator systems which are themselves $r$-polyoids. These generalizations of modules can be considered as $(t+r+1)$-polyoids. However, in order to treat the distributive and associative properties of the semicompositions it is desirable to consider these systems separately.

A $(u, t)$-polymodule is a system $(\Re, \mathfrak{S}, x)$, where

(i) $\mathfrak{R}=\left(R, \circ_{1}, \circ_{2}, \cdots, \circ_{u}\right)$ is a $u$-polyoid,

(ii) $\mathfrak{S}=\left(S,+_{1},+_{2}, \cdots,+_{t}\right)$ is a $t$-polyoid, and

(iii) $x$ is a mapping from a subset of $S \times R$ to $S$. Examples of polymodules are vector spaces, or more generally groups with operators and algebras. Any multiplicative system is an example of a $(1,0)$-polymodule or a $(0,1)$-polymodule. A module, in particular a vector space, is a $(2,1)$-polymodule. Algebras are $(2,2)$-polymodules. 
A $(u[n], t[m])$-polymodule $\mathfrak{\Omega}=(\Re, \mathfrak{S}, x)$ is a $(u+n, t+m)$-polymodule such that $\Re$ is a $(u, n)$-polyring, and $T$ is a $(t, m)$-polyring. In associative algebra is a (0 [2], 0 [2])-polymodule. It can be shown that there are nontrivial realizations of $(u[n], t[m])$-polymodules for all non-negative integers $u, t, m$, and $n$, where the elements are transformations, and also that any $(u, t)$-polymodule can be embedded isomorphically into a $(u[m], t[n])$-polymodule for any positive integers $m$ and $n$.

Every $(u, t)$-polymodule can be considered as a $(u+t+1)$-polyoid. Let $\mathfrak{\Omega}=(\Re, \mathfrak{S}, x)$ be a $(u, t)$-polymodule, where $\mathfrak{R}, \mathfrak{S}$, and $x$ are defined as previously. Let $T=R \cup S,{ }_{i_{i}}, i=1,2, \cdots, u$, and $\circ_{j}, j=1,2, \cdots, t$ be considered as semi-compositions on $T$. Similarly the binary mapping $x$ from a subset of $R \times S$ to $S$ can be considered as a semi-composition on $T$. Hence $\left(T, t_{1}, t_{2}, \cdots, t_{u}, \circ_{1}, \circ_{2}, \cdots, \circ_{\iota}, x\right)$ is a $(u+t+1)$ polyoid.

Another way of studying $(u, t)$-polymodules $(\Re, \mathfrak{S}, x)$ is by identifying the operators (elements of $R$ ) with transformations on the set $S$. The following definitions are needed. The $(u, t)$-polymodules $\mathfrak{I}=(\Re, \mathfrak{S}, x)$ and $\mathfrak{I}^{\prime}=\left(\mathfrak{R}^{\prime}, \mathfrak{S}^{\prime}, x^{\prime}\right)$ are weakly isomorphic if

(i) $\mathfrak{K}^{\prime}$ is a homomorphic image of $\mathfrak{R}$ under a mapping $\theta^{\prime}$,

(ii) $\mathfrak{S}^{\prime}$ is isomorphic to $\subseteq$ under a mapping $\theta^{\prime \prime}$, and

(iii) if $a \in S, b \in R$ such that $a x b$ is defined, then $(a x b) \theta^{\prime \prime}=\left(a \theta^{\prime \prime}\right) x\left(b \theta^{\prime}\right)$. A $(u, t)$-polymodule $\mathfrak{I}=(\Re, \mathfrak{S}, x)$ is a transformation polymodule if the set of elements of $R$ is contained in $S^{1}$, the set of transformations on $S$, and $b \in R, a \in S$ such that $a x b=a b$, the image of $a$ under the mapping $b$.

Every $(u, t)$-polymodule is weakly isomorphic to a subsysystem of $a(u, t)$-transformation polymodule. Let $\mathfrak{I}=(\Re, \mathfrak{S}, x)$ be a $(u, t)$-polymodule.

Case 1. If $r \neq r^{\prime} \in R$ there exists an $s \in S$ such that $s x r$ and $s x r^{\prime}$ are defined and unequal. Let $I_{S}$ be the identity map on $S$. Let $\phi$ be the mapping from $R$ to $S^{1}$ such that $a(b \phi)=a x b$ if $a x b$ is defined, and $a(b \phi)=a$ otherwise, $a \in S, b \in R$. Define the semi-composition $+_{i}^{\prime}, i=$ $1,2, \cdots, u$, to be the semi-composition in $R \phi \subset S^{1}$ where $(a \phi)+{ }_{i}^{\prime}(b \phi)$ is defined if and only if $a+{ }_{i} b$ is defined and $(a \phi)+{ }_{i}^{\prime}(b \phi)=\left(a+{ }_{i} b\right) \phi$. The semi-composition is well-defined since $\phi$ is a $1-1$ map. The system $\mathfrak{I}^{\prime}=\left(\mathfrak{R}^{\prime}, \mathfrak{S}^{\prime}, x^{\prime}\right)$ is weakly isomorphic to $\mathfrak{I}$, where $\mathfrak{R}^{\prime}=\left(R \phi,+_{1}^{\prime},{ }_{2}^{\prime}, \cdots,+_{u}^{\prime}\right)$, and $a x^{\prime} b$ is the image of $a$ under the transformation $b \in T \phi$. For, trivially, $I_{S}$ is an isomorphism from $\mathfrak{S}$ onto $\mathfrak{S}$; $\phi$, by definitions of the semi-compositions in $\Re$, is a homomorphism of $\Re$ onto $\Re^{\prime}$, and $(a x b) I_{s}=$ $a x b=a(b \phi)=\left(a I_{s}\right) x^{\prime}(b \phi)$.

Case 2. Let $\mathfrak{I}^{\prime \prime}=\left(\mathfrak{R}^{\prime \prime}, \mathfrak{S}^{\prime \prime}, x^{\prime \prime}\right)$, where $\mathfrak{R}^{\prime \prime}=\Re, \mathfrak{S}^{\prime \prime}=\left(S \cup R,+{ }_{1}^{\prime \prime}\right.$, $\left.+_{2}^{\prime \prime}, \cdots,+{ }_{t}^{\prime \prime}\right),+_{i}^{\prime \prime}$ is the semi-composition of $\subseteq$ considered in the extended 
set $S \cup R, i=1,2, \cdots, t$, and $x^{\prime \prime}$ is defined as follows: for $a \in S, b \in R$ such that $a x b$ is defined, let $a x b=a x^{\prime \prime} b$, otherwise let $a x^{\prime \prime} b=b$. $\mathfrak{T}^{\prime \prime}$ is clearly a $(u, t)$-polymodule containing a weakly isomorphic image of $\mathfrak{T}$. Further $\mathfrak{I}^{\prime \prime}$ satisfies the condition of Case 1, and the Theorem follows.

Many of the theorems of the previous sections can be generalized. A few of these are stated informally without proof. Given a $(u, t)$ polymodule $\mathfrak{I}=(\Re, \mathfrak{S}, x)$, the conclusions of Theorems 1 and 2 follow for $\Re$ and $\mathfrak{S}$ separately. Further it is possible to a get a weak isomorphism from $\mathfrak{I}$ to $\mathfrak{T}^{*}=\left(\mathfrak{R}^{*}, \mathfrak{S}^{*}, x^{*}\right)$ where

(i) $\mathfrak{T}^{*}$ is a transformation polymodule,

(ii) $\mathfrak{S}^{*}$ is contained in a transformation polyring,

(iii) there is a natural isomorphism from $\mathfrak{S}$ to $\mathfrak{S}^{*}$, and

(iv) there is a homomorphism ('natural') from $\Re$ to $\mathfrak{R}^{*}$. The mapping $x$ can be extended to these larger systems of transformations so that there exists a larger $(u, t)$-polymodule containing a weakly isomorphic image of $\mathfrak{T}$. The corollary of Theorem 1 can be generalized to guarantee the existence of $(u[n], t[m])$-polymodules $\mathfrak{I}=(\mathfrak{R}, \mathfrak{S}, x)$ for any nonnegative integers $m, n, t, u$, so that $\Re$ and $\subseteq$ are $(u, m)$ - and $(t, n)$-polyrings of transformations, respectively.

The concept of a $(u, t)$-polymodule can be further generalized. For example

(i ) $A\left(\left(u_{1}, u_{2}, \cdots, u_{k}\right), t\right)$-polymodule is a system $\left(\Re_{1}, \Re_{2}, \cdots, \Re_{k}\right.$, $\left.\mathfrak{S}, x_{1}, x_{2}, \cdots, x_{k}\right)$, where $\Re_{i}$ is a $u_{i}$-polyoid, $i=1,2, \cdots, k$, $\subseteq$ is a $t$-polyoid, and $x_{i}$ is a mapping from a subset of $S \times R_{i}$ to $S, i=1,2, \cdots, k$.

(ii) $\mathrm{A}\left(u_{k}\left(u_{k-1}\left(\cdots\left(u_{1}, t\right) \cdots\right)\right.\right.$-polymodule is a system $\left(\Re_{1}, \Re_{2}, \cdots\right.$, $\mathfrak{R}_{k}, \mathfrak{S}, x_{1}, x_{2}, \cdots, x_{k}$ ), where $\mathfrak{R}_{i}$ and $\mathfrak{S}$ are defined as in (i), and $x_{i}$ is a mapping from a subset of $\left(R_{i-1} \times R_{i-2} \times \cdots \times R_{1} \times S\right) \times R_{i}$ to $\left(R_{i-1} \times\right.$ $\left.R_{i-2} \times \cdots \times R_{1} \times S\right)$. Many other types of similar systems can also be built up from $t$-polyoids. In fact consider any ordered collecton of symbols representing $t$-polyoids. Then various bracketings and punctuations by commas of these symbols indicate other algebraic systems which may be defined (and which exist). Any such system can be considered a $u$-polyoid for a proper $u$. Generalizations of the previous theorems can be obtained.

\section{REFERENCES}

1. D. W. Blackett, Simple and semi-simple near-rings, Proc. Amer. Math. Soc., 4 (1953), 772-795.

2. Samuel Bourne, The Jacobson radical of a semi-ring, Proc. Nat. Acad. Sci., U.S.A., 6 (163-170).

3. R. H. Bruck, Analogues of the ring of rational integers, Proc. Amer. Math. Soc., 6 (1955), 50-58.

4. N. Jacobson, The Theory of Rings, New York, Amer. Math. Soc. (1943).

5. H. J. Zassenhaus, Über endliche Fastkörper, Abh. Math. Sem. Hamburgischen Univ., 11 (1936), 187-220.

UNIVERSITY OF WATERLOO AND ILLINOIS INSTITUTE OF TEChNOLOGY 


\section{PACIFIC JOURNAL OF MATHEMATICS}

\section{EDITORS}

\author{
David Gilbarg \\ Stanford University \\ Stanford, California \\ F. H. Brownell \\ University of Washington \\ Seattle 5 , Washington
}

\author{
A. L. Whiteman \\ University of Southern California \\ Los Angeles 7, California \\ L. J. PAIGE \\ University of California \\ Los Angeles 24, California
}

\section{ASSOCIATE EDITORS}

\author{
E. F. BECKENBACH \\ T. M. CHERRY \\ D. DERRY
}
E. HEWITT
A. HORN
L. NACHBIN
M. OHTSUKA
H. L. ROYDEN
M. M. SCHIFFER

E. SPANIER

E. G. STRAUS

F. WOLF

\section{SUPPORTING INSTITUTIONS}

\author{
UNIVERSITY OF BRITISH COLUMBIA \\ CALIFORNIA INSTITUTE OF TECHNOLOGY \\ UNIVERSITY OF CALIFORNIA \\ MONTANA STATE UNIVERSITY \\ UNIVERSITY OF NEVADA \\ NEW MEXICO STATE UNIVERSITY \\ OREGON STATE COLLEGE \\ UNIVERSITY OF OREGON \\ OSAKA UNIVERSITY \\ UNIVERSITY OF SOUTHERN CALIFORNIA
}

\author{
STANFORD UNIVERSITY \\ UNIVERSITY OF TOKYO \\ UNIVERSITY OF UTAH \\ WASHINGTON STATE COLLEGE \\ UNIVERSITY OF WASHINGTON

AMERICAN MATHEMATICAL SOCIETY
CALIFORNIA RESEARCH CORPORATION
HUGHES AIRCRAFT COMPANY
SPACE TECHNOLOGY LABORATORIES
NAVAL ORDNANCE TEST STATION

Mathematical papers intended for publication in the Pacific Journal of Mathematics should be typewritten (double spaced), and the author should keep a complete copy. Manuscripts may be sent to any one of the four editors. All other communications to the editors should be addressed to the managing editor, L. J. Paige at the University of California, Los Angeles 24, California. ..

50 reprints per author of each article are furnished free of charge; additional copies may be obtained at cost in multiples of 50 .

The Pacific Journal of Mathematics is published quarterly, in March, June, September, and December. The price per volume (4 numbers) is $\$ 12.00$; single issues, $\$ 3.50$. Back numbers are available. Special price to individual faculty members of supporting institutions and to individual members of the American Mathematical Society: $\$ 4.00$ per volume; single issues, $\$ 1.25$.

Subscriptions, orders for back numbers, and changes of address should be sent to Pacific Journal of Mathematics, 2120 Oxford Street, Berkeley 4, California.

Printed at Kokusai Bunken Insatsusha (International Academic Printing Co., Ltd.), No. $6_{4}$ 2-chome, Fujimi-cho, Chiyoda-ku, Tokyo, Japan.

PUBLISHED BY PACIFIC JOURNAL OF MATHEMATICS, A NON-PROFIT CORPORATION

The Supporting Institutions listed above contribute to the cost of publication of this Journăl, but they are not owners or publishers and have no responsibility for its content or policies. 


\section{Pacific Journal of Mathematics}

\section{Vol. 10, No. $3 \quad$ November, 1960}

Glen Earl Baxter, An analytic problem whose solution follows from a simple

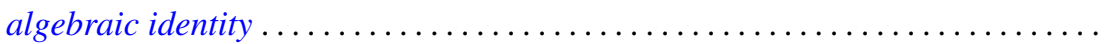

Leonard D. Berkovitz and Melvin Dresher, A multimove infinite game with linear payoff. .

Earl Robert Berkson, Sequel to a paper of A. E. Taylor ......................

Gerald Berman and Robert Jerome Silverman, Embedding of algebraic systems.... 767

Peter Crawley, Lattices whose congruences form a boolean algebra . . . . . ...... 777

Robert E. Edwards, Integral bases in inductive limit spaces . . . . . . . . . . . . . . .

Daniel T. Finkbeiner, II, Irreducible congruence relations on lattices . . . . . . . . . .

William James Firey, Isoperimetric ratios of Reuleaux polygons . . . . . . . . . . . 787

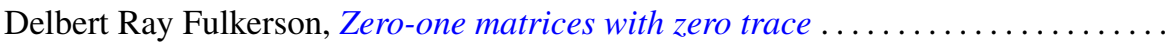

Leon W. Green, A sphere characterization related to Blaschke's conjecture........

Israel (Yitzchak) Nathan Herstein and Erwin Kleinfeld, Lie mappings in

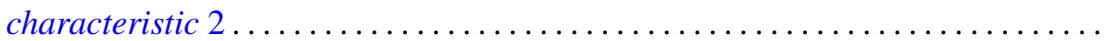

Charles Ray Hobby, A characteristic subgroup of a $p$-group .................

R. K. Juberg, On the Dirichlet problem for certain higher order parabolic

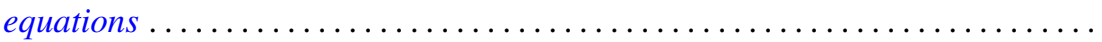

Melvin Katz, Infinitely repeatable games ......................

Emma Lehmer, On Jacobi functions . . . . . . . . . . . . . . . . . . . . . . . . .

D. H. Lehmer, Power character matrices

Henry B. Mann, A refinement of the fundamental theorem on the density of the sum of two sets of integers.

Marvin David Marcus and Roy Westwick, Linear maps on skew symmetric matrices: the invariance of elementary symmetric functions . .

Richard Dean Mayer and Richard Scott Pierce, Boolean algebras with ordered

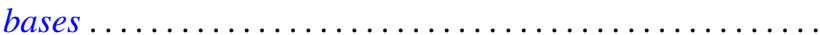

Trevor James McMinn, On the line segments of a convex surface in $E_{3} \ldots$

Frank Albert Raymond, The end point compactification of manifolds ..

Edgar Reich and S. E. Warschawski, On canonical conformal maps of regions of arbitrary connectivity

Marvin Rosenblum, The absolute continuity of Toeplitz's matrices...

Lee Albert Rubel, Maximal means and Tauberian theorems . .

Helmut Heinrich Schaefer, Some spectral properties of positive linear operators

Jeremiah Milton Stark, Minimum problems in the theory of pseudo-conformal transformations and their application to estimation of the curvature of the invariant metric.

Robert Steinberg, The simplicity of certain groups ...

Hisahiro Tamano, On paracompactness. .

Angus E. Taylor, Mittag-Leffler expansions and spectral theory .

Marion Franklin Tinsley, Permanents of cyclic matrices ...... . 